\title{
ANÁLISE SIMULTÂNEA DOS INDICADORES BIOLÓGICOS DE EXPOSIÇÃO AOS SOLVENTES ETILBENZENO, ESTIRENO, TOLUENO E XILENO NA URINA POR CLAE-UV
}

\author{
Priscila A. Yamamoto, Natália V. de Moraes*, Vinicius P. Silva e José S. Lepera \\ Departamento de Princípios Ativos Naturais e Toxicologia, Faculdade de Ciências Farmacêuticas, Universidade Estadual Paulista \\ Júlio de Mesquita Filho, Rodovia Araraquara-Jaú - km 1, s/n, 14800-903 Araraquara - SP, Brasil
}

Recebido em 29/02/2016; aceito em 11/05/2016; publicado na web em 15/07/2016

\begin{abstract}
SIMULTANEOUS ANALYSIS OF BIOLOGICAL INDICATORS OF EXPOSURE TO ETHYLBENZENE, STYRENE, TOLUENE AND XYLENE SOLVENTS IN URINE BY HPLC-UV. A method for simultaneous analysis of phenylglyoxylic (PGA), mandelic (MA), hippuric (HA), orto-, meta- and para-methylhippuric (o-, $m$ - and $p$-MHA) acids in urine, by high performance liquid chromatography with UV detection was developed and validated. The substances are biotransformation products used as biological indicators to assess the exposure of workers, respectively, to ethylbenzene, styrene, toluene and xylene. The urinary metabolites were separated using a C18 column and a mixture of $5 \mathrm{mM}$ phosphate buffer $\mathrm{pH}$ 2.6:acetonitrile (92:8, v/v) as mobile phase. Urine samples were extracted with dichloromethane:ethyl acetate (70:30, v/v). The method used phenacetin as internal standard and was linear in the interval of 100-500 $\mu \mathrm{g} \mathrm{mL}^{-1}$ for PGA and MA and 150-700 $\mu \mathrm{g} \mathrm{mL}{ }^{-1}$ for HA and MHA. The detection limits in $\mu \mathrm{g} \mathrm{mL}{ }^{-1}$, were 19.8 for PGA, 1.7 for MA, 4.1 for HA, 3.9 for $o$-MHA, 3.3 for $m$-MHA and 7.3 for $p$-MHA. Intra- and inter-assay precisions (as relative standard deviation) were all less than $15 \%$ and accuracy (as relative standard error) did not exceed $12.3 \%$. The recovery was higher than $65 \%$ for all metabolites. The developed method will be applied to biological evaluation of workers exposure to these solvents.
\end{abstract}

Keywords: volatile organic compounds; urine; biological monitoring; HPLC-UV.

\section{INTRODUÇÃo}

Os compostos orgânicos etilbenzeno, estireno, tolueno e xileno são amplamente utilizados como solventes em indústrias de plástico, resina, borracha e farmacêutica. ${ }^{1-4}$ Também são constituintes de combustíveis, tintas, vernizes e colas. ${ }^{4-6}$ Trabalhadores expostos a níveis elevados desses solventes podem apresentar efeitos adversos, como irritação (da pele e do trato respiratório), alterações do sistema nervoso central, alterações funcionais nos nervos ótico e óptico. ${ }^{5}$ Portanto, a monitorização da exposição, tanto ambiental quanto biológica, são atividades complementares e fundamentais na prevenção de tais efeitos. A monitorização ambiental avalia o risco à saúde do indivíduo exposto através da concentração dos solventes na zona respiratória, comparando-a aos Limites de Exposição Ocupacional. Por sua vez, a monitorização biológica pode avaliar a dose interna do composto para verificar se a exposição do trabalhador está em níveis aceitáveis, por comparação com o respectivo Limite para o Indicador Biológico utilizado. Para os solventes, os marcadores biológicos (ou indicadores biológicos) podem ser a própria substância ou os seus metabólitos presentes em matrizes biológicas. ${ }^{7}$

A principal via de absorção do etilbenzeno, estireno, tolueno e xileno é a via respiratória e a eliminação ocorre, principalmente, através dos metabólitos urinários. ${ }^{5}$ Os principais metabólitos do etilbenzeno e do estireno são o ácido mandélico (AM) e o ácido fenilglioxílico (AFG). No entanto, esses metabólitos não são indicadores específicos, pois também são formados quando há exposição a outras substâncias, por exemplo, estirenoglicol, óxido de estireno, fenilglicol, acetofenona, fenilglicina. ${ }^{5} \mathrm{O}$ principal metabólito urinário do orto-, meta- e para-xileno são os ácidos orto-, meta- e para-metil hipúricos $(o-, m$ - e p-AMH), respectivamente, os quais são completamente excretados dentro de 1 ou 2 dias depois da exposição, fato que limita a utilização desses metabólitos como indicadores biológicos apenas a exposições recentes. ${ }^{8}$ Para o tolueno, o principal metabólito é o ácido hipúrico

*e-mail: nmoraes@fcfar.unesp.br
(AH). Os valores de referência (VR) e os índices biológicos máximos permitidos (IBMP) desses indicadores biológicos estabelecidos pela Norma Regulamentadora $\mathrm{n}^{\circ} 7$ (NR-7) e os BEIs ${ }^{\circledR}$ - Biological Exposure Indices propostos pela ACGIH (American Conference of Governmental Industrial Hygienists) - são apresentados na Tabela 1.,9

Este estudo teve por objetivo desenvolver e validar um método simples para análise simultânea de AFG, AM, AH, $o$-AMH, $m$-AMH e $p$-AMH em urina por cromatografia líquida de alta eficiência com detecção por ultravioleta (CLAE-UV).

Esta Nota Técnica foi apresentada no $8^{\circ}$ Congresso de Extensão Universitária da UNESP e seu resumo expandido encontra-se disponível online. ${ }^{10}$

\section{PARTE EXPERIMENTAL}

\section{Reagentes}

Os padrões do ácido fenilglioxílico (AFG, 97\%), ácido mandélico (AM, 99\%), ácido hipúrico (AH, 98\%), ácido metil hipúrico (orto-AMH, meta-AMH e para-AMH, 98\%) e fenacetina (padrão interno) foram obtidos da Sigma Aldrich (St Louis, MO, EUA). Os solventes metanol (J.T. Baker, Ecatepec, Estado do México, México), acetato de etila (Sigma Aldrich, St. Louis, MO, EUA), diclorometano (J. T. Baker, Center Valley, PA, EUA) e acetonitrila (J.T. Baker, Center Valley, PA, EUA) utilizados foram grau CLAE. O ácido $o$-fosfórico 85\% (Synth, Diadema, Brasil), fosfato de sódio monobásico (Synth, Diadema, Brasil) e ácido clorídrico 37\% (Qhemis, Brasil) são reagentes de grau analítico. A água utilizada em todas as soluções foi obtida através de um sistema de purificação Milli-Q Plus (Millipore, Belford, MA, EUA).

As soluções-padrão estoque foram preparadas separadamente dissolvendo os padrões em metanol para obter a concentração de $14 \mathrm{mg} \mathrm{mL}^{-1}$ de AFG e AM e $20 \mathrm{mg} \mathrm{mL}^{-1}$ de AH, $o$-AMH, $m$-AMH e $p$-AMH. O padrão interno fenacetina foi preparado na concentração de $2 \mathrm{mg} \mathrm{mL}^{-1}$ em metanol. Os padrões de calibração foram 
Tabela 1. Indicadores biológicos de exposição ao etilbenzeno, estireno, tolueno e xileno na urina

\begin{tabular}{|c|c|c|c|c|}
\hline Solvente & Indicador biológico & VR & IBMP & $B E I^{\circledast}$ \\
\hline \multirow[t]{2}{*}{ Etilbenzeno } & Ácido mandélico & - & $1,5 \mathrm{~g} \mathrm{~g}^{-1}$ creat. & $0,7 \mathrm{~g} \mathrm{~g}^{-1}$ creat. $^{\mathrm{a}}$ \\
\hline & Ácido fenilglioxílico & & - & \\
\hline \multirow[t]{2}{*}{ Estireno } & Ácido mandélico & - & $0,8 \mathrm{~g} \mathrm{~g}^{-1}$ creat. & $0,4 \mathrm{~g} \mathrm{~g}^{-1}$ creat. $^{\mathrm{a}}$ \\
\hline & Ácido fenilglioxílico & & $0,24 \mathrm{~g} \mathrm{~g}^{-1}$ creat. & \\
\hline Tolueno & Ácido hipúrico & Até $1,5 \mathrm{~g} \mathrm{~g}^{-1}$ creat. & 2,5 $\mathrm{g} \mathrm{g}^{-1}$ creat. & - \\
\hline Xileno & Ácidos metil hipúricos & - & $1,5 \mathrm{~g} \mathrm{~g}^{-1}$ creat. & $1,5 \mathrm{~g} \mathrm{~g}^{-1}$ creat. \\
\hline
\end{tabular}

creat.: creatinina; VR: valor de referência; IBMP: índice biológico máximo permitido; $B E I^{\circledR}:$ Biological Exposure Index. ${ }^{\mathrm{A}} \mathrm{A}$ ACGIH propõe a soma de ambos metabólitos urinários como indicador biológico.

preparados diluindo as soluções-padrão estoque em metanol para obter concentrações variando de 400 a $2000 \mu \mathrm{g} \mathrm{mL} \mathrm{m}^{-1}$ para AFG e AM $\left(400,800,1200,1400\right.$ e $\left.2000 \mu \mathrm{gL}^{-1}\right)$ e de 600 a $2800 \mu \mathrm{g}$ $\mathrm{mL}^{-1}$ para AH, $o$-AMH, $m$-AMH e $p$-AMH $(600,1000,1400,1960$, $\left.2800 \mu \mathrm{g} \mathrm{mL}^{-1}\right)$.

\section{Instrumentação}

A análise dos metabólitos foi realizada por CLAE-UV utilizando um cromatógrafo líquido de alta eficiência Perkin Elmer, composto de um sistema binário de bombas Flexar LC Pump, degaseificador e detector Flexar UV/VIS. A coluna utilizada foi Brownlee C18 SSP $(100 \times 3,0 \mathrm{~mm} ; 2,7 \mu \mathrm{m})$ da Perkin Elmer. O sistema de cromatografia foi controlado pelo programa Chromera UV/Vis (Perkin Elmer). A mistura de tampão fosfato $5 \mathrm{mM}(\mathrm{pH} 2,6)$ :acetonitrila $(92: 8 \mathrm{v} / \mathrm{v})$ foi utilizada como fase móvel, em fluxo de $0,6 \mathrm{~mL} \mathrm{~min}^{-1}$. A detecção por ultravioleta foi realizada a $254 \mathrm{~nm}$ nos primeiros 3,0 min e a $225 \mathrm{~nm}$ até o final da análise para todos os metabólitos.

\section{Preparo das amostras}

Alíquotas de $200 \mu \mathrm{L}$ de urina e $20 \mu \mathrm{L}$ de solução de fenacetina $2 \mathrm{mg} \mathrm{mL}^{-1}$ (padrão interno) foram adicionadas em um microtubo de $2 \mathrm{~mL}$. A amostra foi acidificada com $20 \mu \mathrm{L}$ de ácido clorídrico 1 $\mathrm{M}$ e submetida à extração líquido-líquido com $1 \mathrm{~mL}$ da mistura de diclorometano:acetato de etila (70:30, v/v). As amostras foram agitadas por 30 min e posteriormente centrifugadas a $10.600 \times g$ por 10 min, a $4{ }^{\circ} \mathrm{C}$. Seiscentos microlitros da fase orgânica foram evaporados a secura em banho seco a $70{ }^{\circ} \mathrm{C}$. O resíduo foi reconstituído com $600 \mu \mathrm{L}$ de fase móvel, filtrado em membrana de $0,45 \mu \mathrm{m}$ (Analítica, São Paulo, Brasil) e uma alíquota de $20 \mu \mathrm{L}$ do filtrado foi injetada no sistema CLAE-UV.

\section{Curvas de calibração e linearidade}

Três curvas de calibração para análise de AFG, AM, AH, o-AMH, $m$-AMH e $p$-AMH foram preparadas em alíquotas de $200 \mu \mathrm{L}$ de urina branco coletada de indivíduos não expostos aos solventes precursores dos metabólitos em estudo enriquecidas com $50 \mu \mathrm{L}$ de cada padrão de calibração. As curvas de calibração incluíram a análise de amostra branco e amostras com as seguintes concentrações finais de 100, 200, 300, 350 e $500 \mu \mathrm{g} \mathrm{mL}^{-1}$ para AFG e AM; 150, 250, 350, 490 e $700 \mu \mathrm{g} \mathrm{mL}^{-1}$ para AH, $o$-AMH, $m$-AMH e $p$-AMH em urina. As amostras da curva de calibração foram processadas e analisadas conforme descrito previamente.

Controles de qualidade foram preparados enriquecendo amostras de urina branco com três diferentes níveis de concentração (100, 200 e $420 \mu \mathrm{g} \mathrm{mL}^{-1}$ para AFG e AM; 140, 250 e $588 \mu \mathrm{g} \mathrm{mL}^{-1}$ para $\mathrm{AH}$, $o$-AMH, $m$-AMH e $p$-AMH).

\section{Limites de detecção (LD) e quantificação (LQ)}

Os limites de detecção e de quantificação foram obtidos como recomenda o National Institute for Occupational Safety and Health (NIOSH) ${ }^{11}$ Em resumo, foram preparados padrões de calibração de baixa concentração em metanol variando de 100 a $2000 \mu \mathrm{g} \mathrm{mL} \mathrm{m}^{-1}$ para AFG, 6 a $120 \mu \mathrm{g} \mathrm{mL}^{-1}$ para AM, 4 a $80 \mu \mathrm{g} \mathrm{mL}^{-1}$ para $\mathrm{AH}$ e 30 a 600 $\mu \mathrm{g} \mathrm{mL}^{-1}$ para $o$-AMH, $m$-AMH e $p$-AMH. Três curvas de calibração foram preparadas em alíquotas de $200 \mu \mathrm{L}$ de urina branco coletada de indivíduos não expostos enriquecidas com $50 \mu \mathrm{L}$ de cada padrão de calibração de baixa concentração. A triplicata da curva de calibração de baixa concentração foi utilizada para obter a equação de regressão linear (Equação 1) e o erro padrão da regressão (Sy) (Equação 2). O erro padrão da regressão e o coeficiente angular da equação de regressão linear foram usados para o cálculo do limite de detecção (Equação 3). O limite de quantificação foi calculado como 3,33 vezes o limite de detecção (Equação 4).

$$
\begin{gathered}
Y=a X+b \\
S y=\sqrt{\frac{\sum\left(\hat{Y}_{i}-Y_{i}\right)^{2}}{(N-2)}} \\
L D=\frac{3 \times S y}{\text { coeficiente angular }} \\
L Q=3,33 \times L D
\end{gathered}
$$

onde: $\hat{Y}_{i}$ é a resposta predita de cada concentração do analito $(X) ; Y_{i}$ é a resposta obtida $(Y) ; N$ é o número de padrões de calibração de baixa concentração $(N=5)$

\section{Recuperação}

A recuperação foi determinada enriquecendo alíquotas de $200 \mu \mathrm{L}$ de urina branco com concentrações finais na urina de 200 e $350 \mu \mathrm{g}$ $\mathrm{mL}^{-1}$ de AFG e AM, 250 e $490 \mu \mathrm{g} \mathrm{mL}^{-1}$ de AH, $o$-AMH, $m$-AMH e p-AMH, e $20 \mu \mathrm{L}$ de solução de fenacetina $\left(2 \mathrm{mg} \mathrm{mL}^{-1}\right)$. As amostras $(n=5)$ foram extraídas como descrito anteriormente. A recuperação foi calculada comparando a área dos picos das amostras extraídas conforme o protocolo analítico com a área obtida a partir das soluções-padrão de mesma concentração não submetidas ao processo de extração, sendo esta última considerada $100 \%$.

\section{Precisão e exatidão}

A precisão e exatidão intra e interensaios foram investigadas utilizando os controles de qualidade de baixa (CQB), média (CQM) 
e alta (CQA) concentração analisados em quintuplicata em 3 dias diferentes. A precisão foi expressa como desvio padrão relativo (coeficiente de variação, CV) (Equação 5) e a exatidão como erro padrão relativo (EPR) (Equação 6).

$$
C V=\frac{\text { desvio padrão } \times 100}{\text { concentração média experimental }}
$$

$$
E P R=\frac{(\text { concentração média experimental }- \text { valor nominal }) \times 100}{\text { valor nominal }}
$$

\section{RESULTADOS E DISCUSSÃO}

No presente estudo, foi desenvolvido e validado um método utilizando CLAE-UV capaz de analisar simultaneamente os indicadores biológicos de exposição aos solventes etilbenzeno, estireno, tolueno e xileno. Vários métodos para analisar AFG, AM, AH, $o$-AMH, $m$-AMH e $p$-AMH na urina foram descritos na literatura, porém poucos são capazes de analisar todos os metabólitos simultaneamente..$^{4,6,12-14} \mathrm{Os}$ métodos que utilizam cromatografia gasosa acoplada à espectrometria de massas têm sido amplamente utilizados. Entretanto, tais métodos exigem um processo de derivatização durante o preparo da amostra., ${ }^{43}$ Entre os métodos que utilizam a cromatografia líquida para a análise simultânea dos mesmos analitos, há aquele em que a separação dos analitos foi observada apenas após 50 minutos, ${ }^{12}$ aquele que utiliza grande volume de amostra $(2 \mathrm{~mL})$ e solventes orgânicos ou não utilizam padrão interno. ${ }^{6,12,14}$

A Figura 1 apresenta os cromatogramas obtidos com o presente método. Os metabólitos foram completamente separados em menos de 30 minutos, com exceção do $m$-AMH e $p$ - $\mathrm{AMH}$, os quais coeluem (Figura 1A). No entanto, a separação completa desses isômeros não se faz necessária para a presente aplicação, uma vez que é recomendado o uso da soma dos isômeros do AMH como indicador biológico para a exposição a xilenos. ${ }^{5,9} \mathrm{O}$ método mostrou-se seletivo, uma vez que não foram observados picos interferentes no tempo de retenção dos metabólitos, como pode ser observado ao comparar o cromatograma de uma urina branco (Figura 1C) com o cromatograma da solução-padrão (Figura 1B). Para que um método seja considerado seletivo, as respostas de picos interferentes próximo ao tempo de retenção do analito devem ser inferiores a $20 \%$ da resposta do analito nas amostras de LIQ.

A análise de uma urina branco proveniente de indivíduo não exposto ao tolueno apresenta um pico de AH (Figura 1C), que foi encontrado na urina humana provavelmente devido ao consumo de alimentos que contêm ácido benzoico ou benzoato como conservador. ${ }^{5,15-16}$ Esse conservador está presente em refrigerantes, sucos de frutas, margarina, molho de tomate, enlatados. ${ }^{17-19}$ Um estudo identificou os refrigerantes como a principal fonte de benzoato no Brasil, representando mais de $80 \%$ da ingestão diária do conservador. ${ }^{17} \mathrm{O}$ ácido benzoico ou benzoato é metabolizado no fígado e nos rins formando o AH. ${ }^{20} \mathrm{~A}$ alimentação e a variação interindividual no metabolismo do ácido benzoico influenciam diretamente na quantidade basal de AH presente na urina. A ACGIH, atualmente, não recomenda o uso do AH como indicador biológico para a exposição a tolueno, pois a elevada quantidade basal de $\mathrm{AH}$ pode dificultar a interpretação no limite de exposição atualmente recomendado para o tolueno, de 75 $\mathrm{mg} \mathrm{m}^{-3}$. No entanto, no Brasil a legislação ainda prevê o uso do $\mathrm{AH}$ como indicador biológico de exposição ao tolueno. ${ }^{9}$ Vale ressaltar que a legislação brasileira referente aos indicadores biológicos de exposição ao tolueno não é atualizada desde $1994 .{ }^{9}$

Trabalhos anteriores sugerem realizar a análise de AFG a $254 \mathrm{~nm}$ pois o metabólito apresenta uma resposta melhor do que a $225 \mathrm{~nm} .{ }^{12,21}$ Nos cromatogramas apresentados na Figura 2 é possível observar a diferença na resposta do AFG quando analisado a $225 \mathrm{~nm}$ (Figura 2A)
A) Urina de individuo năo exposto enriquecida com soluçăo-padrăo

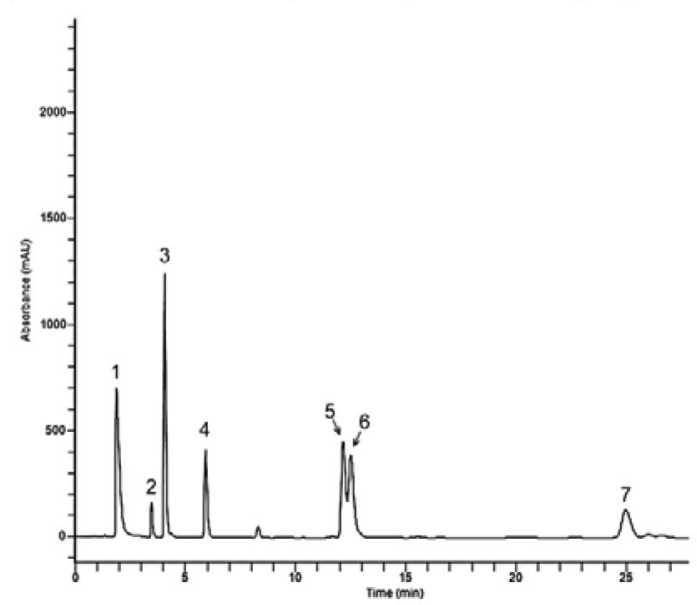

B) Solução-padrão

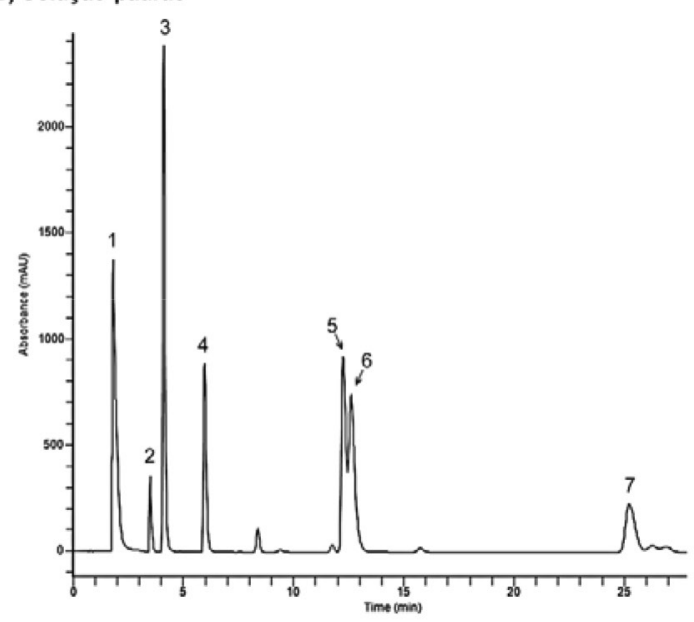

C) Urina branco

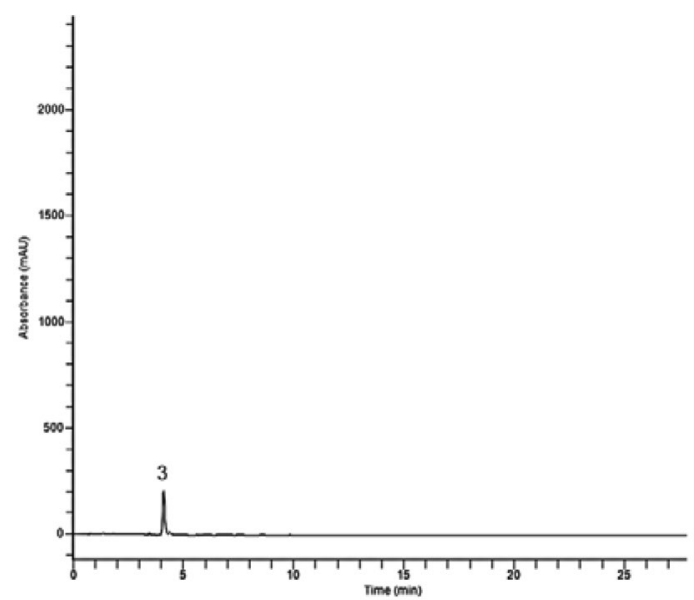

Figura 1. Cromatogramas dos seis metabólitos obtidos com o presente método (a) Urina não exposta enriquecida com solução-padrão (300 $\mu \mathrm{g} m L^{-1}$ de AFG e AM e $350 \mu \mathrm{g} m L^{-1}$ de AH, o-AMH, m-AMH e p-AMH), (b) Solução-padrão (300 $\mu \mathrm{g} m L^{-1}$ de AFG e AM e $350 \mu \mathrm{g} m L^{-1}$ de AH, o-AMH, m-AMH e p-AMH) e (c) Urina branco [1. $A F G$, 2. AM, 3. AH, 4. o-AMH, 5. m-AMH, 6. p-AMH, 7. Fenacetina (P.I.)]

e a $254 \mathrm{~nm}$ (Figura 2B). Portanto, o presente método propõe a análise do AFG a $254 \mathrm{~nm}$ e os demais metabólitos a $225 \mathrm{~nm}$.

A curva de calibração foi gerada através do gráfico da razão entre área do analito/área do padrão interno vs. a concentração do analito. As curvas de calibração para o AFG, AM, AH, $o$-AMH, $m$-AMH e 
A) $\lambda=225 \mathrm{~nm}$

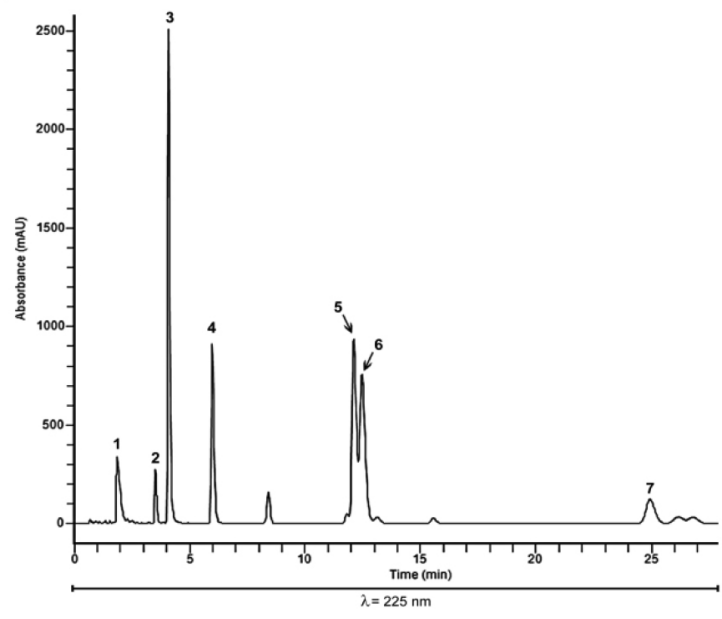

B) $\lambda=254 \mathrm{~nm}$ e $225 \mathrm{~nm}$

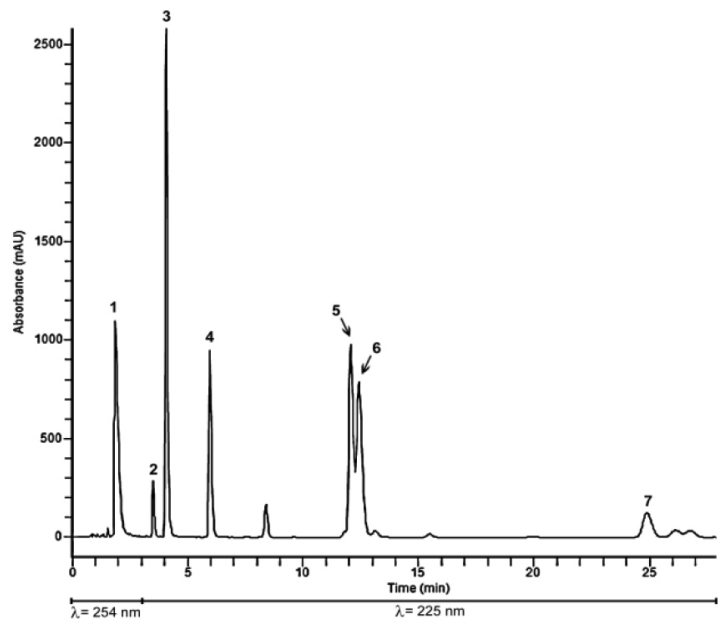

Figura 2. Cromatogramas utilizando diferentes comprimentos de onda (a) Análise utilizando apenas $\lambda=225 \mathrm{~nm}$ e (b) Análise utilizando $\lambda=254 \mathrm{~nm}$ nos primeiros 3 min e $\lambda=225 \mathrm{~nm}$ a partir dos 3 min até o final da corrida [1. $A F G$, 2. AM, 3. AH, 4. o-AMH, 5. m-AMH, 6. p-AMH, 7. Fenacetina (P.I.)]
p-AMH em urina enriquecida foram lineares nos intervalos de concentração estudados (Tabela 2).

O ensaio de recuperação está relacionado a eficiência da extração e foi superior a $65 \%$ para todos os metabólitos e o padrão interno e não variou em função da concentração (Tabela 3). Embora a porcentagem de recuperação próxima a $100 \%$ tanto para o analito como para o padrão interno seja desejável, porcentagens inferiores são admitidas desde que a recuperação seja reprodutível entre diferentes concentrações do analito. Os limites de detecção e quantificação foram calculados a partir da equação de regressão linear e do erro padrão da regressão. Os limites de detecção obtidos para AFG, AM, AH, $o$-AMH, $m$-AMH e $p$-AMH foram de $19,83 \mu \mathrm{g} \mathrm{mL}^{-1}, 1,73 \mu \mathrm{g} \mathrm{mL} \mathrm{m}^{-1}$, $4,12 \mu \mathrm{g} \mathrm{mL} L^{-1}, 3,93 \mu \mathrm{g} \mathrm{mL} L^{-1}, 3,29 \mu \mathrm{g} \mathrm{mL}{ }^{-1}$ e 7,29 $\mu \mathrm{g} \mathrm{mL}^{-1}$, respectivamente (Tabela 2) e são compatíveis com a aplicação do método para avaliação biológica da exposição ocupacional aos solventes estireno, etilbenzeno, xilenos e tolueno.

Alíquotas de urina coletada de indivíduo não exposto foram enriquecidas com AFG, AM, AH, $o-\mathrm{AMH}, m-\mathrm{AMH}$ e $p$-AMH e analisadas em quintuplicata para determinar a precisão e exatidão intra e interensaio. Todos os metabólitos apresentaram coeficiente de variação menor que $15 \%$. A exatidão, expressa como erro padrão relativo, apresentou variação menor que $12,3 \%$ para os três níveis de concentração testados (Tabela 4).

\section{CONCLUSÃO}

Foi desenvolvido e validado um método utilizando CLAE-UV capaz de analisar simultaneamente os indicadores biológicos AM, AFG, AH, $o$-AMH, $m$-AMH e $p$-AMH em urina. A sensibilidade, precisão e exatidão do método mostraram-se compatíveis com a sua aplicação na monitorização biológica da exposição ocupacional aos solventes etilbenzeno, estireno, tolueno e xileno conforme recomendado pela NR-7 e ACGIH.

\section{AGRADECIMENTOS}

Os autores agradecem a Pró-Reitoria de Extensão Universitária da UNESP (PROEX) e a Coordenadoria de Saúde e Segurança do Trabalhador e Sustentabilidade Ambiental (COSTSA).

Tabela 2. Parâmetros da curva de calibração, limite de detecção (LD) e limite de quantificação (LQ) dos metabólitos

\begin{tabular}{|c|c|c|c|c|c|}
\hline Metabólito & $\begin{array}{c}\text { Curva de Calibração } \\
\left.(\mu \mathrm{g} \mathrm{mL})^{-1}\right)\end{array}$ & Equação & $\mathrm{r}^{2}$ & $\mathrm{LD}\left(\mu \mathrm{g} \mathrm{mL} \mathrm{m}^{-1}\right)$ & $\mathrm{LQ}\left(\mu \mathrm{g} \mathrm{mL} \mathrm{m}^{-1}\right)$ \\
\hline AFG & $100-500$ & $y=0,0082 x-0,4975$ & 0,9874 & 19,83 & 66,03 \\
\hline $\mathrm{AM}$ & $100-500$ & $y=0,0009 x-0,0039$ & 0,9964 & 1,73 & 5,76 \\
\hline $\mathrm{AH}$ & $150-700$ & $y=0,0054 x+0,0062$ & 0,9977 & 4,12 & 13,71 \\
\hline$o-\mathrm{AMH}$ & $150-700$ & $y=0,0032 x-0,0578$ & 0,9950 & 3,93 & 13,08 \\
\hline$m$-AMH & $150-700$ & $y=0,0053 x-0,0847$ & 0,9963 & 3,29 & 10,95 \\
\hline$p$-АMH & $150-700$ & $y=0,0056 x-0,0948$ & 0,9971 & 7,29 & 24,26 \\
\hline
\end{tabular}

$\mathrm{r}^{2}$ : coeficiente de correlação linear.

Tabela 3. Recuperação do AFG, AM, AH, $o$-AMH, $m$-AMH, $p$-AMH e fenacetina adicionados em urina não exposta

\begin{tabular}{lcccc}
\hline Metabólito & Concentração $\left(\mu \mathrm{g} \mathrm{mL} \mathrm{m}^{-1}\right)$ & Recuperação $(\%)$ & Concentração $\left.(\mu \mathrm{g} \mathrm{mL})^{-1}\right)$ & Recuperação $(\%)$ \\
\hline AFG & 200 & $65,7 \pm 3,7$ & 350 & $77,7 \pm 5,1$ \\
AH & 200 & $72,3 \pm 1,8$ & 350 & $74,6 \pm 1,7$ \\
AH & 250 & $68,0 \pm 0,9$ & 490 & $65,5 \pm 3,9$ \\
$o-$ AMH & 250 & $75,5 \pm 0,8$ & 490 & $73,7 \pm 4,1$ \\
$m$-AMH & 250 & $78,5 \pm 0,7$ & 490 & $77,6 \pm 6,9$ \\
$p$-AMH & 250 & $80,6 \pm 0,9$ & 490 & $77,3 \pm 6,5$ \\
Fenacetina & 200 & $90,0 \pm 1,6$ & 200 & $80,6 \pm 7,7$ \\
\hline
\end{tabular}


Tabela 4. Precisão e exatidão intra e interensaio do método de análise de AFG, AM, AH e AMH em urina por CLAE-UV (n=5)

\begin{tabular}{|c|c|c|c|c|c|}
\hline \multirow{2}{*}{ Metabólito } & \multirow{2}{*}{ Conc. $\left(\mu \mathrm{g} \mathrm{mL}^{-1}\right)$} & \multicolumn{2}{|c|}{ Precisão (CV, \%) } & \multicolumn{2}{|c|}{ Exatidão (EPR, \%) } \\
\hline & & Intraensaio & Interensaio $^{\mathrm{a}}$ & Intraensaio & Interensaio $^{\mathrm{a}}$ \\
\hline \multirow{3}{*}{ AFG } & 100 & 7,13 & 14,64 & $-0,14$ & 2,63 \\
\hline & 200 & 14,25 & 13,34 & $-8,61$ & $-7,51$ \\
\hline & 420 & 10,88 & 9,31 & $-5,66$ & $-7,32$ \\
\hline \multirow{3}{*}{ AM } & 100 & 3,29 & 6,24 & 6,80 & 4,80 \\
\hline & 200 & 1,54 & 3,14 & 9,60 & 9,26 \\
\hline & 420 & 2,68 & 5,14 & 5,76 & 7,51 \\
\hline \multirow{3}{*}{$\mathrm{AH}$} & 140 & 2,52 & 8,57 & $-11,11$ & $-12,24$ \\
\hline & 250 & 2,28 & 2,83 & $-0,12$ & $-0,58$ \\
\hline & 588 & 2,80 & 6,00 & 4,31 & 0,12 \\
\hline \multirow{3}{*}{$o-\mathrm{AMH}$} & 140 & 2,68 & 7,46 & 7,23 & 6,65 \\
\hline & 250 & 1,41 & 2,80 & $-1,99$ & $-2,52$ \\
\hline & 588 & 2,49 & 6,29 & 5,84 & 0,89 \\
\hline \multirow{3}{*}{$m$-AMH } & 140 & 2,09 & 6,36 & 1,02 & 0,04 \\
\hline & 250 & 1,80 & 2,64 & $-3,49$ & $-4,21$ \\
\hline & 588 & 2,56 & 5,79 & 2,61 & $-1,63$ \\
\hline \multirow{3}{*}{$p$-AMH } & 140 & 2,01 & 6,79 & 2,15 & 1,78 \\
\hline & 250 & 1,60 & 3,45 & 3,08 & 0,82 \\
\hline & 588 & 4,18 & 6,31 & 0,10 & $-4,58$ \\
\hline
\end{tabular}

CV: coeficiente de variação $[\mathrm{CV}=($ desvio padrão/concentração média experimental $)$ x 100]; EPR: erro padrão relativo $\{\mathrm{EPR}=[(\mathrm{concentração} \mathrm{média} \mathrm{experi-}$ mental - valor nominal)/valor nominal] x 100\}. aPrecisão e exatidão interensaio foram avaliadas em quintuplicata durante 3 dias.

\section{REFERÊNCIAS}

1. Crandall, M. S.; Am. Ind. Hyg. Assoc. J. 1981, 42, 499.

2. Seedorff, L.; Olsen, E.; Ann. Occup. Hyg. 1990, 34, 371.

3. Truchon, G.; Brodeur, J.; Gerin, M.; J. Anal. Toxicol. 1990, 14, 227.

4. Szúcs, S.; Tóth, L.; Legoza, J.; Sárváry, A.; Ádány, R.; Arch. Toxicol. 2002, 76, 560 .

5. 2011 TLVs and BEIs with 7th Edition Documentation 2011 [Cd-room], ACGIH: Cincinatti, 2011.

6. Onchoi, C.; Kongtip, P.; Yoosook, W.; Chantanajul, S.; Southeast Asian J. Trop. Med. Public Health 2008, 39, 1164.

7. Teass, A. W.; Biagini, R. E.; DeBord, D. G.; Hull, R D. Em Manual of Analytical Methods; Eller, P. M., ed.; NIOSH: Cincinnati, 1998, cap. F.

8. http://www.atsdr.cdc.gov/ToxProfiles/tp.asp?id=296\&tid=53, acessada em Julho 2016.

9. http://www.mtps.gov.br/images/Documentos/SST/NR/NR7.pdf, acessada em Julho 2016

10. Esta Nota Técnica foi apresentada no $8^{\circ}$ Congresso de Extensão Universitária da UNESP e seu resumo expandido encontra-se disponível no endereço http://200.145.6.205/index.php/congressoextensao/8congre ssoextensao/paper/viewFile/322./182
11. Kennedy, E. R.; Fischbach, T. J.; Song, R.; Eller, P. M.; Shulman, S. A.; Guidelines for Air Sampling and Analytical Method Development and Evaluation, NIOSH: Cincinnati, 1995, apêndice 3.

12. Ogata, M.; Taguchi, T.; Int. Arch. Occup. Environ. Health 1987, 59, 263.

13. Ohashi, Y.; Mamiya, T.; Mitani, K.; Wang, B.; Takigawa, T.; Kira, S.; Kataoka, H.; Anal. Chim. Acta 2006, 566, 167.

14. Antunes, M. V.; Patuzzi, A. L. M.; Linden, R.; Quim. Nova 2008, 31, 1865 .

15. Ogawa, M.; Suzuki, Y.; Endo, Y.; Kawamoto, T.; Kayama, F.; Ind. Health. 2011, 49, 195.

16. Antunes, M. V.; Niederauer, C. G.; Linden, R.; Clin. Biochem. 2013, 46, 1276.

17. Tfouni, S. A. V.; Toledo, M. C. F.; Food Addit. Contam. 2002, 19, 647.

18. Gonzalez, K. C.; Sagebin, F. R.; Oliveira, P. G.; Glock, L.; Thiesen, F. V.; Ciência \& Saúde Coletiva 2010, 15, 1637.

19. Alves, A. C.; Rangel, C. V.; Pratte-Santos, R.; Revista Sapientia 2012, 11,34 .

20. Badenhorst, C. P. S.; Erasmus, E.; Van Der Sluis, R.; Nortje, C.; Van Dijk, A. A.; Drug Metab. Rev. 2014, 46, 343.

21. Ogata, M.; Sugihara, R.; Int. Arch. Occup. Environ. Health 1978, 42, 11. 